\title{
Large quasineutral electron velocity oscillations in radial expansion of an ionizing plasma
}

A R Karimov, M Y Yu and Lennart Stenflo

\section{Linköping University Post Print}

N.B.: When citing this work, cite the original article.

Original Publication:

A R Karimov, M Y Yu and Lennart Stenflo, Large quasineutral electron velocity oscillations in radial expansion of an ionizing plasma, 2012, Physics of Plasmas, (19), 9, 092118. http://dx.doi.org/10.1063/1.4754865

Copyright: American Institute of Physics (AIP) http://www.aip.org/

Postprint available at: Linköping University Electronic Press http://urn.kb.se/resolve?urn=urn:nbn:se:liu:diva-85093 


\title{
Large quasineutral electron velocity oscillations in radial expansion of an ionizing plasma
}

\author{
A. R. Karimov, ${ }^{1, a)}$ M. Y. Yu, ${ }^{2,3, b)}$ and L. Stenflo ${ }^{4}$ \\ ${ }_{1}^{1}$ Institute for High Temperatures, Russian Academy of Sciences, Izhorskaya 13/19, Moscow 127412, Russia \\ ${ }^{2}$ Department of Physics, Institute for Fusion Theory and Simulation, Zhejiang University, 310027 Hangzhou, \\ China \\ ${ }^{3}$ Institut für Theoretische Physik I, Ruhr-Universität Bochum, D-44780 Bochum, Germany \\ ${ }^{4}$ Department of Physics, Linköping University, SE-58183 Linköping, Sweden
}

(Received 13 July 2012; accepted 12 September 2012; published online 28 September 2012)

Radial expansion of an ionizing gas or plasma cylinder into vacuum is investigated. An exact model for the evolution of the density and velocity fields of the electrons, ions, and neutrals, including the effect of photo and electron-impact ionization on the flow characteristics is developed and solutions obtained. A quasineutral nonlinear electrostatic mode involving rapid oscillations in the electron velocity but not in the density can occur in the expanding plasma. The mode turns out to be almost unaffected by weak ionization. (C) 2012 American Institute of Physics. [http://dx.doi.org/10.1063/1.4754865]

\section{INTRODUCTION}

Expansion of an ionizing gas into vacuum remains a topic of much interest because of its relevance in many areas of research and applications, including laser-plasma interaction, astrophysical jets and clouds, phenomena in ionospheric and space plasmas, laboratory electrical discharges, etc. ${ }^{1-4}$ The process can be accompanied or even dominated by condensation, chemical reactions, ionization, recombination, etc., and can involve several time and space scales that are of similar, or differ by several orders of, magnitude. To investigate such flows, perturbation methods based on a priori timescale separation or fixing are inapplicable and one has to take into account all possible timescales of the system of interest..$^{5-13}$

Most existing works on the flow of reacting or ionizing gases invoke linear, or nonlinear but small amplitude, perturbations around a steady state. ${ }^{7-13}$ However, for phenomena initially far from equilibrium, especially in the interaction of intense laser with matter and in astrophysical or nuclear explosions, such perturbation approaches are not applicable since the field variations can be larger, or even much larger, than the background fields. In this case, a fully nonlinear treatment of the governing equations is called for. In the present paper, we examine how ionization influences the evolution of expanding plasma layers by allowing for large field variations. A nonperturbative approach based on the method of separation of variables shall be used. ${ }^{14,15}$ In particular, we consider the effect of ionization ${ }^{16}$ on the flow dynamics and largeamplitude oscillations in a homogeneous expanding cylindrical plasma. ${ }^{15,17}$ It is found that in the expanding plasma, there can exist a quasineutral nonlinear electrostatic mode with fast oscillations in the electron velocity that is not accompanied by density oscillations. Because of the quasineutral character of ionization, weak ionization does not significantly affect the expansion and the mode.

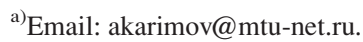

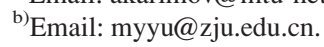

\section{GOVERNING EQUATIONS}

We shall consider radially symmetric expansion ${ }^{15}$ of an unmagnetized cylindrical plasma consisting of neutral atoms, cold ions, and electrons, accounting for electron-impact ionization or photo ionization in the presence of an intense external light source such as a sun or a laser beam. The domain of the expansion is infinite, i.e., there are no wall or other boundary effects. At $t=0$, the system is located in $0<r<a$ and its density is given by

$$
n_{0 s}(r)=N_{0 s},
$$

where the subscripts $s=e, i$, and $n$ denote electron, ion, and neutral gas quantities, respectively. The constants $a$ and $N_{0 s}$ define the initial density profile of the plasma. For convenience, we assume $N_{0 e}=N_{0 i}=N_{0 n}=N_{0}$, although initially nonneutral systems can also be included. Elastic collisions among the charged particles, such as that of electron-electron, electron-ion, etc, are usually ignorable in low-temperature plasmas, which are usually weakly ionized and the plasma density is low.

We can write the governing fluid equations in the dimensionless forms

$$
\begin{gathered}
\partial_{t}\left(r n_{s}\right)+\partial_{r}\left(r n_{s} v_{s}\right)=r W_{s}, \\
\partial_{t} v_{s}+v_{s} \partial_{r} v_{s}=\mu_{s} E+W_{s} v_{n}, \\
\partial_{r}(r E)=r\left(n_{i}-n_{e}\right),
\end{gathered}
$$

where $\mu_{e}=-1, \mu_{i}=m_{e} / m_{i}$, and $\mu_{n}=0$. The radial coordinate $r$ and the time $t$ are normalized by $a$ and $\omega_{p e}^{-1}$ $=\left(m_{e} / 4 \pi N_{0} e^{2}\right)^{1 / 2}$, respectively, where $e$ and $m_{e}$ are the charge and mass of the electrons. The densities $n_{s}$, velocities $v_{s}$, and the electric field $E$ have been normalized by $N_{0}, a \omega_{p e}$, and $4 \pi e N_{0} a$, respectively, and $W_{s}$ is the normalized rate of ionization.

Significant photoionization of a neutral gas occurs in the presence of sufficiently intense radiation. Depending on the properties of the radiation and the gas, it can appear in many 
forms, including single and multiple photon ionization, and avalanche ionization or optical breakdown, etc., as well combinations of them. For photoionization by an intense (say of intensity $I \sim 10^{14} \mathrm{~W} / \mathrm{cm}^{2}$ ) radiation source, the normalized rate for the simplest photoionization mode can be written as ${ }^{2}$

$$
W_{e}=W_{i}=-W_{n}=\frac{I \sigma_{\mathrm{ph}}(\omega)}{\hbar \omega \omega_{p e}} n_{n}=\nu_{\mathrm{ph}} n_{n},
$$

where $\sigma_{\mathrm{ph}}$ is the photoionization cross section of the neutral gas atom at the frequency $\omega$ of the radiation. We have assumed $a \omega_{p e} \gg c \gg v_{\text {osc }}$, where $v_{\text {osc }}=e E_{0} / \omega m_{e}$ is the maximum oscillation speed of an electron in the light-wave electric field $E_{0}=(8 \pi I / c)^{1 / 2}$ and $c$ is the light speed, so that radiation driven plasma dynamics can be neglected. If $\left|v_{s}\right| \sim v_{\text {osc }}$, an external harmonic electric field, say $E_{\text {ext }} \sim \sin (\omega t)$ of the radiation, should be added to the self-consistent fields. As a result, the plasma will also be driven by the imposed oscillating field as well as the resulting ponderomotive force and thus gain additional energy. However, this effect requires a separate treatment and is beyond the scope of the present work.

Electron-impact ionization of a gas occurs if the latter is of sufficiently (but not too) high temperature (say, $>16 \mathrm{eV}$ for the common gases, but the ionization rate decreases for too high temperatures since the collision cross section becomes smaller, see Eq. (8) below.) For electron-impact ionization, the normalized ionization rates can be written as ${ }^{2}$

$$
W_{e}=W_{i}=-W_{n}=\frac{k_{i} N_{0}}{\omega_{p e}} n_{n} n_{e}=\nu_{\mathrm{coll}} n_{n} n_{e}
$$

where $k_{i}$ is the ionization constant. Thus, the inelastic ionization processes are characterized by the rates $\nu_{\mathrm{ph}}$ and $\nu_{\text {coll }}$. We note that the photo ionization and electron-impact ionization nonlinearly affect the particle continuity and momentum equations in different manners.

The effect of elastic inter-particle collisions on the dynamics of the plasma expansion and oscillations is negligible since the plasma component is rarefied and such collisions do not affect the ionization process. ${ }^{1,2}$ The condition for neglect of elastic collisions can be written as

$$
\frac{\nu_{s n}}{\omega_{p e}}\left|\frac{v_{s}-v_{n}}{v_{s}}\right| \ll 1, \quad s=e, i,
$$

where $\nu_{s n}$ is the collision frequency of the $s(=i, e)$ particles with neutrals. This condition is well satisfied for rarefied partially ionized plasmas except perhaps in phenomena with very long time scales, in which case the cumulated dissipative effects may play a role in the behavior of the system. The rate of particle production by ionization is also assumed to be much larger than that of particle loss by recombination, or $W_{e} \gg W_{L}$, where $W_{L}$ denotes particle loss due to electronion volume recombination. For impact ionization by thermal electrons in a quasi-neutral plasma, we have ${ }^{18}$

$$
W_{e}=\frac{n_{e} n_{n} \bar{v}_{e} \sigma_{e}}{N_{0} \omega_{p e}}\left(U_{i}+2 k T_{e}\right) \exp \left(-U_{i} / k T_{e}\right),
$$

where $U_{i}$ is the ionization potential, $\sigma_{e}$ is the characteristic ionization cross section, $\bar{v}_{e}=\left(8 k T_{e} / \pi m_{e}\right)^{1 / 2}, T_{e}$ is the characteristic electron temperature, and $k$ is Boltzmann's constant. For the recombination, we have $\mathrm{e}^{18}$

$$
W_{L}=\frac{4}{3} \frac{\pi^{2} r_{0}^{5} \bar{v}_{e} n_{e}^{3}}{N_{0} \omega_{p e}}
$$

where $r_{0}=2 e^{2} / 3 k T_{e}$ is the Coulomb radius. The condition $W_{e} \gg W_{L}$ then becomes

$$
\frac{\sigma_{e}}{\sigma_{c}}\left(\frac{U_{i}}{k T_{e}}+2\right) \exp \left(-\frac{U_{i}}{k T_{e}}\right) \gg \frac{4 \pi}{3} \frac{n_{e}}{n_{n}} \frac{n_{e}}{n_{c}},
$$

where $\sigma_{c}=\pi r_{0}^{2}$ and $n_{c}=1 / r_{0}^{3}$. Analogously, for photo ionization, the corresponding condition is

$$
\frac{\sigma_{e}}{\sigma_{c}} \frac{I}{\hbar \omega n_{e} \bar{v}_{e}} \gg \frac{4 \pi}{3} \frac{n_{e}}{n_{n}} \frac{n_{e}}{n_{c}},
$$

so that Eqs. (7), (10), and (11) determine the conditions under which particle production by ionization is dominant. They are easily satisfied for low-temperature plasmas. Note that since photo ionization involves photon-atom interaction, it is usually much faster than the collisional processes.

\section{EVOLUTION OF THE PLASMA}

In order to solve Eqs. (2)-(4) without perturbation, we look for self-similar flow structures in the form

$$
v_{s}=r V_{s}(t), \quad n_{s}=N_{s}(t),
$$

which corresponds to spatially uniform electron, ion, and neutral densities and a given velocity profile that has a linear $r$ dependence. ${ }^{14,15} \mathrm{We}$ shall see that with this simple structure, the space-time dependence of the collective plasma dynamics and the full nonlinearity of the given system can be included without approximation in obtaining the solution, and the evolution of the system can still be complex and physically interesting.

Substitution of Eq. (12) into Eq. (2) yields

$$
d_{t} N_{s}+2 N_{s} V_{s}=W_{s},
$$

where $s=e, i$, and $n$, and $W_{s}$ is given by Eq. (5) for photo ionization and Eq. (6) for impact ionization. The Poisson's equation (4) can now be written as

$$
E=\left(N_{i}-N_{e}\right) r / 2 .
$$

Inserting Eqs. (12) and (14) into Eq. (3), we obtain

$$
N_{s} d_{t} V_{s}+N_{s} V_{s}^{2}=\left(\mu_{s} / 2\right) N_{s}\left(N_{i}-N_{e}\right)+W_{s} V_{n},
$$

completing set of coupled nonlinear ordinary differential equations describing the evolving plasma in the presence of ionization. The equation differs from that in Ref. 15 by the presence in Eqs. (13) and (15) of the nonlinear source terms. As we shall see, these differences can lead to interesting new physical characteristics in the dynamics of the expanding plasma.

It is instructive to first consider plasma expansion without ionization by setting $W_{s}=0$ in Eqs. (2) and (3). To avoid 
singular behavior, we shall restrict our investigation to initially expanding plasmas, namely $V_{s}(0)>0$ for $s=e$, $i$, and $n$. We also assume $\mu_{i}=10^{-5}$ (roughly hydrogen plasma). For the purpose of comparison, we shall use initial conditions close to that in Ref. 15 , namely $V_{i}(0)=V_{n}(0)=10^{-3}$, $V_{e}(0)=1.1 \times 10^{-3}$, and $N_{e}(0)=N_{i}(0)=N_{n}(0)=1$. Note also that although the initial densities of the plasma species are the same, the initial velocities $V_{e}(0), V_{i}(0)$, and $V_{n}(0)$ are nonzero and not equal, so that charge separation (and plasma oscillations) is not precluded.

The evolution of the velocity and density parameters is shown in Fig. 1. For clarity, in the figures, all velocities are normalized by $V_{n}(0)$. As expected because of the expansion, both the electron and ion velocities decrease. Moreover, because of the large ion mass, the decrease in the ion velocity remains monotonic, but high-frequency nonlinear electron velocity oscillations initiated by the small difference between $V_{e}(0)$ and $V_{i}(0)$ appear. If there is no initial velocity difference, solutions similar to that of a neutral gas can be found. In fact, if we set $V_{e}(0)=V_{i}(0)=V_{n}(0)=V(0)$, $N_{e}(0)=N_{i}(0)=N_{n}(0)=N(0)$, and $\mu_{e}=\mu_{i}=0$, we obtain from Eqs. (13) and (15)

$$
V(t)=V(0) /(1+V(0) t), \quad N(t)=N(0) /(1+V(0) t)^{2},
$$

which as expected describe the evolution of an expanding neutral gas.

Equation (16) can be considered as the basic flow expansion structure. Comparing with Eq. (15), one can see that the latter (or the results shown in Fig. 1) corresponds to a dynamic transformation of the basic structure (Eq. (16)). That is, the nonlinear inertial flow dominates the expansion and its flow structure is preserved even after the nonperturbative inclusion of the electrodynamic and, as shall be seen below, ionization effects.

\section{EFFECT OF IONIZATION}

We now consider the dynamics of an expanding plasma in the presence of ionization. With photo ionization, the set of six ordinary differential Eqs. (13) and (15) are numerically integrated. The results are shown in Fig. 2 for photo ionization with $\nu_{\mathrm{ph}}=10^{-3}$ and $\nu_{\mathrm{ph}}=10^{-2}$. As can be seen in Figs. 2(a) and 2(b), for weak ionization, the behavior of the charged species is similar to that in the absence of ionization, indicating that the effect of the latter is negligible. On the other hand, peaks in the electron and ion density and velocity components can appear for higher ionization rates, as can be seen in Figs. 2(c) and 2(d). This is because the increase in the density and momentum of the charged particles due to ionization can temporally dominate over the reduction due to the expansion. As expected, the density and velocity of the neutrals now decrease faster. This anomalous phenomenon appears only if the ionization rate is within the range $10^{-3}<\nu_{\mathrm{ph}}<10^{-2}$. If the ionization rate is too large, accumulation of the charged particles would dominate over the expansion and thermal effects should be taken into consideration. Fig. 2 also shows that increasing the ionization rate $\nu_{\mathrm{ph}}$ tends to decrease the magnitude of the electron velocity oscillations, which is a direct consequence of flux conservation.

The results for an expanding plasma including electronimpact ionization are shown in Fig. 3 for $\nu_{\text {coll }}=10^{-3}$ and $\nu_{\text {coll }}=10^{-2}$. The monotonic increase in the electron and ion densities with time as well as with $\nu_{\text {coll }}$ reflects the effect of impact ionization. However, because of the plasma expansion and thus decrease of the plasma energy density, the amplitude and frequency of the oscillations in $V_{e}$ decrease with time. As with photo ionization, we can see that the flux conservation leads to reduction of the magnitude of the electron velocity oscillations when $\nu_{\text {coll }}$ is increased. Figs. 3(a) and 3(b) show that for small $\nu_{\text {coll }}$, the results for the electrons and ions are nearly identical to that without ionization. That is weak ionization not only does not destroy the plasma quasineutrality but also does not affect the expansion and the electron oscillations. This can be expected since ionization increases the electron and ion densities in exactly the same manner both in space and time. Similar to the case with photo ionization, Figs. 3(c) and 3(d) show that anomalous peaks in the density and velocity components can also appear in the range $10^{-3}<\nu_{\text {coll }}<10^{-2}$, for similar reasons.
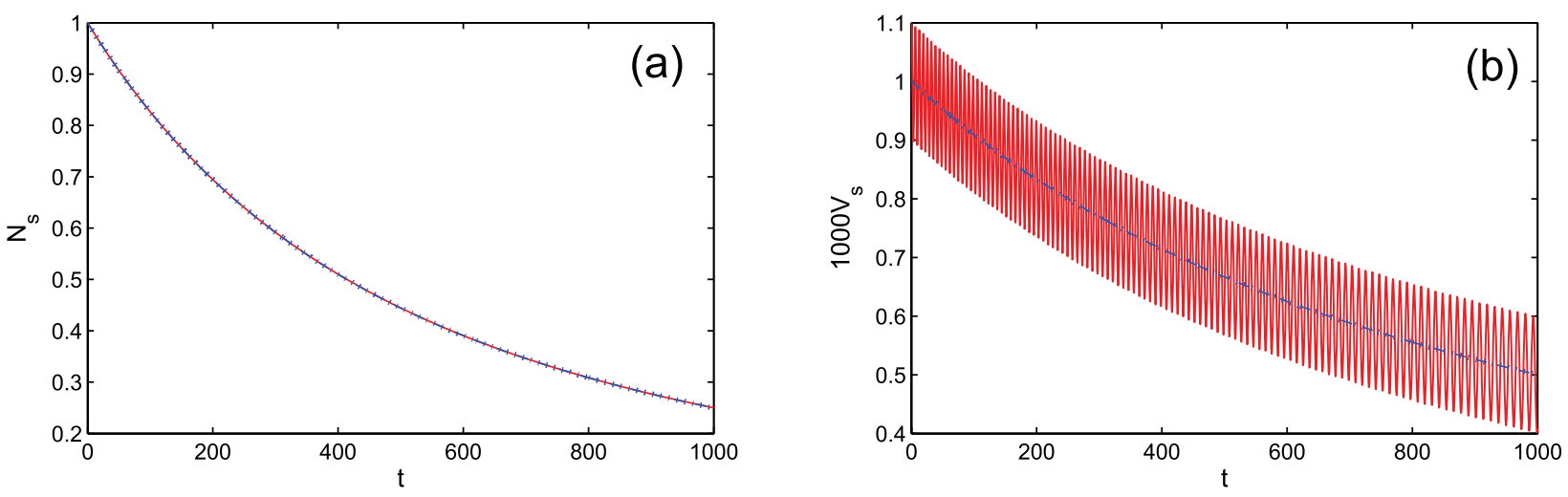

FIG. 1. Evolution of the plasma without ionization. (a) The electron, ion, and neutral-particle density components are given by the red solid, blue dashed, and green dotted curves, respectively. (b) The electron, ion, and neutral-particle radial velocity components are given by the red solid, blue dashed, and green dotted curves, respectively. The three density curves, as well as the ion and neutral velocities curves, overlap, and the electron density oscillates rapidly. That is, the plasma remains quasi neutral despite the rapid oscillations in the electron velocity. 

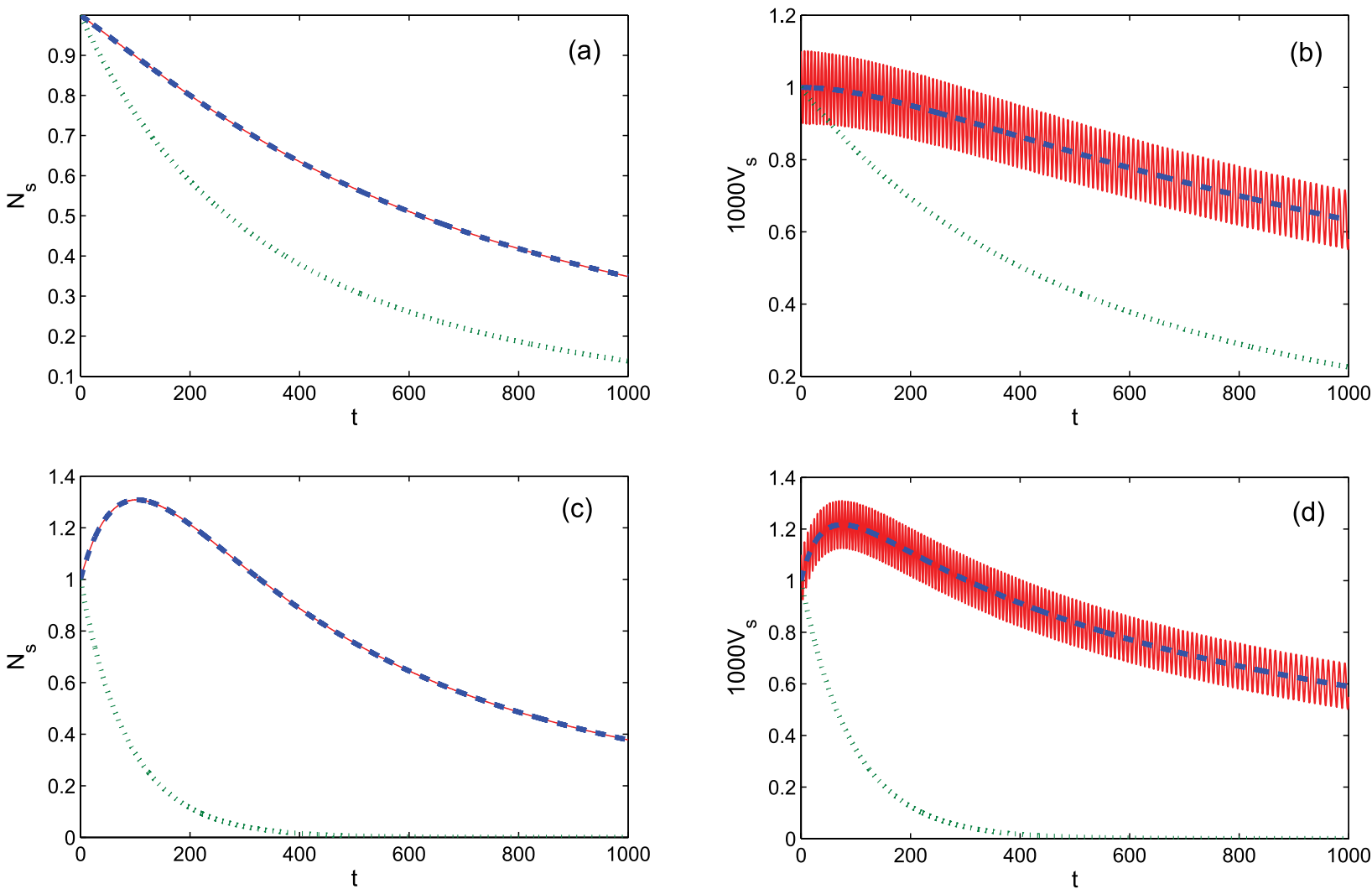

FIG. 2. Evolution of the plasma under photo ionization. (a) and (b) for $\nu_{\mathrm{ph}}=10^{-3}$. (c) and (d) for $\nu_{\mathrm{ph}}=10^{-2}$. The legend is the same as in Fig. 1. The electron and ion density curves overlap. The plasma remains quasi neutral since photo ionization increases the electron and ion densities in the same manner.
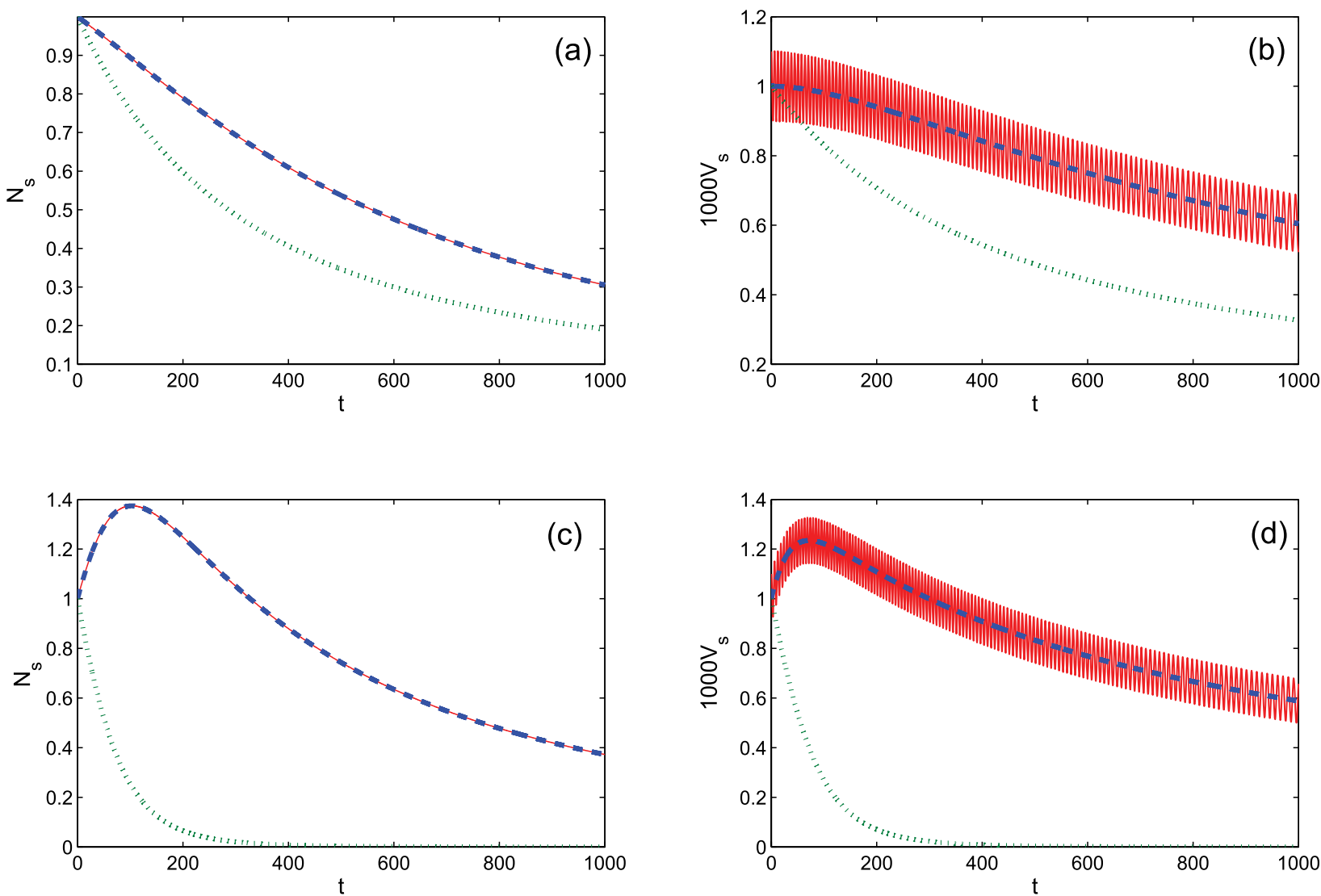

FIG. 3. Evolution of the plasma under electron impact ionization. (a) and (b) For $\nu_{\text {coll }}=10^{-3}$. (c) and (d) for $\nu_{\text {coll }}=10^{-2}$. The legend is the same as in Fig. 1 . The electron and ion density curves overlap. The plasma is again quasi neutral since impact ionization also affects the electron and ion densities in the same manner. Note that for small ionization rates $\left(\nu_{\mathrm{ph}}=10^{-2}\right.$ and $\left.\nu_{\mathrm{coll}}=10^{-2}\right)$, the densities and velocities of the charged particles are similar to that in the absence of ionization. 


\section{CONCLUSION}

We have considered the effect of ionization on the radial expansion and nonlinear electron velocity oscillations of a partially ionized cylindrical plasma by first finding a spatial structure for which the space and time dependence of the flow parameters can be separated. The structure corresponds to uniform densities and linear spatial velocity profiles for the electron and ion flows. Photo ionization and electron impact ionization are considered.

Our results show that fully nonlinear electron oscillations with properties quite different from that of the well known linear electron plasma (Langmuir) oscillations can occur. In particular, the expansion always remains quasi neutral: the highly nonlinear oscillations of the electron velocity take place without electron density variation, and there is no shock or solitary wave formation. This can be attributed to the fact the temporal behavior of the electron density in the expansion depends on time integrals of the electron velocity [see Eq. (13)], so that the oscillations in the latter do not appear in the density. This turns out to be a common property of the type of expansion and nonlinear oscillations considered here, which takes place because of symmetry breaking (with respect to the phase relationship among the physical quantities involved in the linear and weakly nonlinear electron plasma waves) associated with the present mode structure. For the same reason, processes such as ionization, which involves mainly density increase of the charged particles, do not significantly affect the nonlinear oscillations since the plasma remains quasineutral and ionization affects the electron and ion densities in exactly the same manner. This property is in strong contrast with that of the nonlinear plasma oscillations without plasma expansion, where many of the linear plasma wave properties are retained. ${ }^{6,7,16}$ For the problem considered here, the dynamics of the expansion predominates over the oscillations even when the latter is of large amplitude. In the presence of electron-ion collisions, ignored here, one can expect that the electron velocity oscillations will be gradually damped since the oscillation energy can be transferred by electron-ion collisions to the ion motion. The results here indicate that in the presence of expansion, the other linear waves, or normal modes, in plasmas can also behave very differently (in the frequency, dispersion, phase relationship among the physical parameters involved, as well as other properties) from their well known linear counterparts, so that they should perhaps be considered as different modes. This conclusion may be helpful in analyzing unusual phenomena in astrophysics and laserplasma interaction.

\section{ACKNOWLEDGMENTS}

This work was supported by the MoSTC (2011GB105000) and the NNSFC (10835003).

${ }^{1}$ A. V. Gurevich and L. V. Pariiskaya, Reviews of Plasma Physics (Consultants Bureau, New York, 1986), Vol. 10.

${ }^{2}$ Ya. B. Zel'dovich and Yu. P. Raizer, Physics of Shock Waves and HighTemperature Hydrodynamic Phenomena (Dover, Mineola, New York, 2002).

${ }^{3}$ P. Mora, Phys. Rev. Lett. 90, 185002 (2003).

${ }^{4}$ W. Yu, L. Cao, M. Y. Yu, H. Cai, H. Xu, X. Yang, A. Lei, K. A. Tanaka, and R. Kodama, Laser Part. Beams 27, 109 (2009).

${ }^{5}$ B. I. Dovudov, Zh. Eksp. Teor. Fiz. 6, 463 (1936).

${ }^{6}$ A. I. Akhiezer, I. A. Akhiezer, R. V. Polovin, A. G. Sitenko, and K. N Stepanov, Plasma Electrodynamics (Pergamon, Oxford, 1975), Vol. 1.

${ }^{7}$ R. C. Davidson, Methods in Nonlinear Plasma Theory (Academic, New York, 1972).

${ }^{8}$ P. K. Shukla and A. A. Mamun, Introduction to Dusty Plasma Physics (IOP, London, 2002).

${ }^{9}$ M. Chaudhuri, S. A. Khrapak, and G. E. Morfill, Phys. Plasmas 15, 053703 (2008)

${ }^{10}$ O. S. Vaulina, S. V. Vladimirov, A. Yu. Repin, and J. Goree, Phys. Plasmas 13, 012111 (2006).

${ }^{11}$ M. Chaudhuri, S. A. Khrapak, R. Kompaneets, and G. E. Morfill, IEEE Plasma Sci. 38, 818 (2010)

${ }^{12}$ S. A. Khrapak, A. V. Ivlev, and G. E. Morfill, Phys. Plasmas 17, 042107 (2010).

${ }^{13}$ M. Chaudhuri, R. Kompaneets, and G. E. Morfill, Phys. Plasmas 17, 063705 (2010).

${ }^{14}$ A. R. Karimov, L. Stenflo, and M. Y. Yu, Phys. Plasmas 16, 062313 (2009).

${ }^{15}$ A. R. Karimov, L. Stenflo, and M. Y. Yu, Phys. Lett. A 375, 2629 (2011).

${ }^{16}$ A. R. Karimov and V. A. Shcheglov, Phys. Plasmas 7, 1050 (2000).

${ }^{17}$ A. R. Karimov, Phys. Scr. 65, 356 (2002).

${ }^{18}$ Yu. P. Raizer, Gas Discharge Physics (Springer, New York, 1991). 